FOLIA

Amazónica

Revista del Instituto de Investigaciones

de la Amazonía Peruana

\title{
ESTUDIO DE VIABILIDAD Y CONSERVACIÓN DE SEMILLAS DE CINCO ESPECIES VEGETALES AMAZÓNICAS COMO BASE PARA LA CREACIÓN DE UN BANCO DE GERMOPLASMA EN EL MUNICIPIO DE LETICIA, AMAZONAS, COLOMBIA
}

\author{
Astrid HERNÁNDEZ-CORONADO ${ }^{1,2}$, Astrid RODRÍGUEZ ${ }^{1,2}$, Adrián CANDRE ${ }^{1}$, \\ Ismenia PINTO $^{1}$, Elio MIRAÑA ${ }^{1}$, Ana Milena BERNILLA ${ }^{1}$ \\ 1 Servicio Nacional para el Aprendizaje-SENA \\ 2 Grupo de investigación Arara. dendrobaf@hotmail.com, asrodriguezg@sena.edu.co
}

\section{RESUMEN}

Como parte del proceso para el establecimiento de un banco de germoplasma de especies amazónicas maderables y no maderables que contribuyen a la seguridad alimentaria del municipio de Leticia, Amazonas (Colombia), fue realizada la caracterización de once especies vegetales que consistió en la definición de usos, períodos de fructificación y experiencias de propagación, durante el período de junio a octubre de 2017; para avanzar con el análisis de factores que afectan directamente el comportamiento al almacenamiento de germoplasma (semillas), se seleccionaron cinco especies vegetales que se encontraron en cosecha durante el periodo de septiembre a noviembre de 2017, Carapa guianensis, Oenocarpus bataua, Bactris gasipaes, Scleronema praecox y Socratea exorrhiza, a los que se les realizó: ubicación de árboles semilleros, recolección, limpieza, secado previo y determinación del contenido de humedad a las semillas. Como resultados se obtuvo el porcentaje de pureza, contenidos de humedad, secado y almacenamiento de germoplasma. Se realizaron pruebas de germinación en vivero, con diferentes sustratos para determinar su viabilidad antes y después del almacenamiento, mostrando resultados positivos de germinación para las semillas sembradas sin tratamiento de secado y pérdida de viabilidad de las semillas por la disminución del contenido de humedad (semillas recalcitrantes).

PALABRAS CLAVE: Banco de germoplasma, especie vegetal, propagación, Amazonas, viabilidad. 


\title{
VIABILITY STUDY AND CONSERVATION OF SEEDS OF FIVE AMAZON VEGETABLE SPECIES, AS A BASE FOR THE CREATION OF A GERMPLASM BANK, IN THE MUNICIPALITY OF LETICIA, AMAZONAS, COLOMBIA.
}

\begin{abstract}
As part of the process for the establishment of a germplasm bank of timber, nontimber Amazonian species that contribute to the food security of the region, the characterization of eleven plant species was carried out; consisting of definition of uses, fruiting periods and experiences of propagation, during the period from June to October 2017; To advance with the analysis of factors that directly affect the storage behavior of germplasm (seeds), five plant species were selected that were harvested during the period from September to November 2017, Carapa guianensis, Oenocarpus bataua, Bactris gasipaes, Scleronema praecox and Socratea exorrhiza, to which were made: location of seed trees, harvesting, cleaning, previous drying and determination of the moisture content of the seeds. The species Scleronema praecox had high moisture content, during the previous drying it was observed a rapid dehydration and contamination by fungi. Germination tests were carried out in the nursery, with different substrates to determine their viability before and after storage, showing positive germination results for the seeds sown without drying treatment and loss of viability of the seeds due to the decrease in moisture content (seeds recalcitrant).

KEYWORDS: Germplasm bank, plant species, propagation, Amazon, viability.
\end{abstract}




\section{INTRODUCCIÓN}

Dentro de las estrategias de conservación, se considera como una actividad importante el almacenamiento de semillas en bancos de germoplasma para su mantenimiento, preservación y propagación, especialmente para especies que se encuentran amenazadas y su hábitat natural esté ampliamente transformado, por lo que se requiere asegurar la conservación de las especies (Cárdenas et al., 2015). Los bancos de germoplasma tienen como objetivo general la conservación a largo plazo y la accesibilidad del germoplasma vegetal. Dentro de las operaciones básicas de un banco de germoplasma de semillas incluyen la colecta, el procesamiento, la conservación, la regeneración y la distribución del germoplasma (Rao et al., 2007). Sin embargo, es importante recopilar el conocimiento acerca de las especies antes de iniciar estas operaciones, teniendo en cuenta que la información es limitada para las especies vegetales, específicamente para el tratamiento de las semillas de especies tropicales (Willan, 1991).

En esta investigación, se presentan las actividades realizadas con el semillero de investigación Amazonas Verde, perteneciente el Grupo de Investigación Arara del Centro para la biodiversidad y Turismo del Amazonas, del Servicio Nacional para el Aprendizaje-SENA, en las primeras etapas hacia la meta de establecer un banco de germoplasma de especies amazónicas maderables, no maderables y que contribuyan a la seguridad alimentaria de la región, especialmente de las comunidades indígenas y agricultores de Leticia.

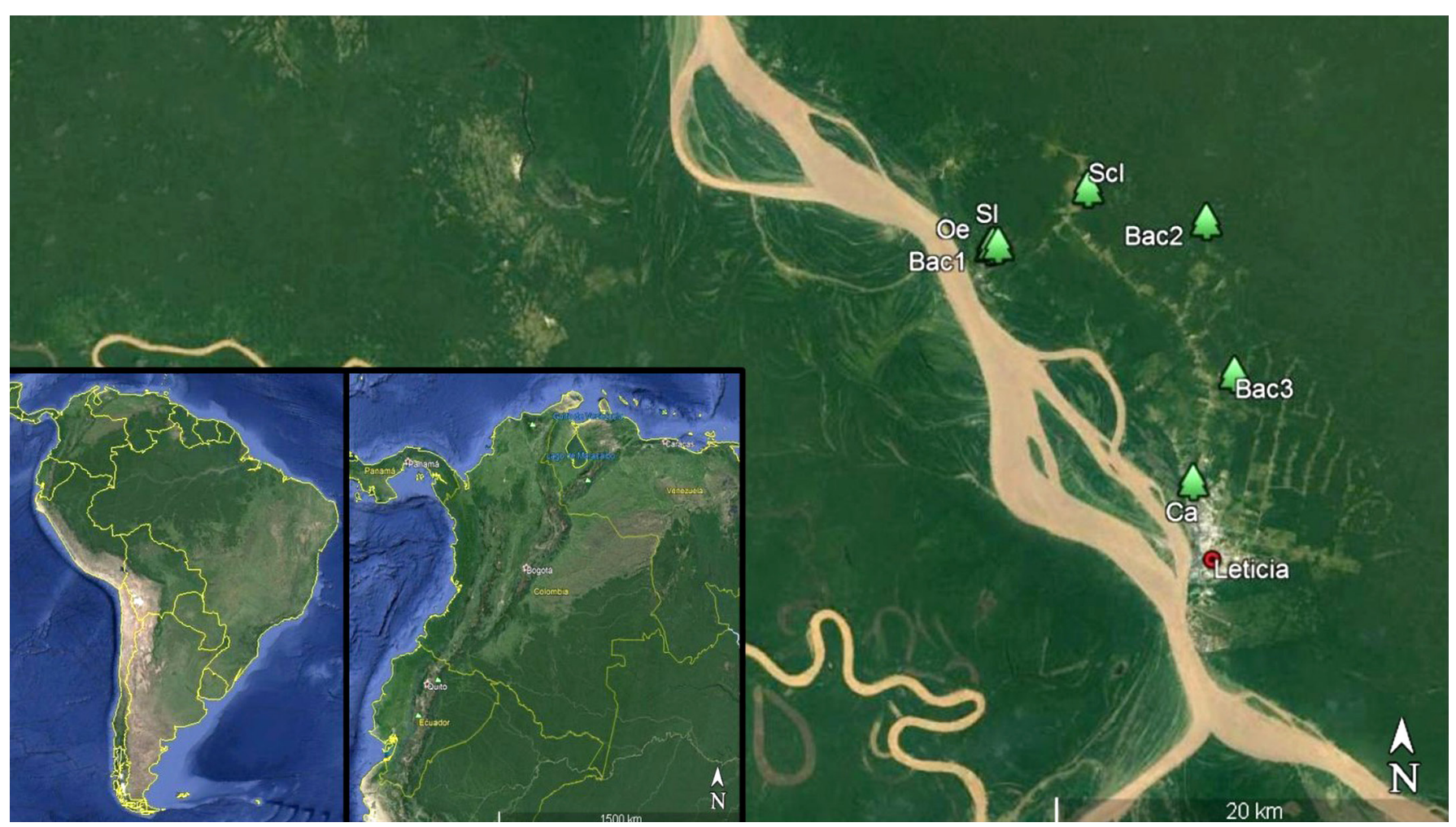

Figura 1. Localización geográfica de los sitios de colecta de semillas en el Municipio de Leticia, Departamento de Amazonas. Bac (Bactris gasipaes), Oe (Oenocarpus bataua), SI (Socratea exhorriza), Scl (Scleronema praecox), Ca (Carapa guianensis). 


\section{METODOLOGÍA}

\section{ÁREA DE ESTUDIO}

El Municipio de Leticia, está situado sobre la margen izquierda del río Amazonas (4²12'55" Latitud Sur y 69 $56^{\prime} 26^{\prime \prime}$ Longitud Oeste). La cabecera Municipal se localiza entre 0 y $80 \mathrm{~m}$ de altitud sobre el nivel del mar. Dentro del municipio existen 27 comunidades indígenas en el sector rural, pertenecientes a las etnias tikuna, cocama, yagua, uitoto y bora principalmente (Alcaldía de Leticia, 2002; Alcaldía de Leticia, 2018).

La búsqueda de árboles semilleros y la recolección se realizó tanto en zona urbana como rural. En total se visitaron 7 localidades (Comunidad de Nazareth, Comunidad Bora Km 17, Comunidad Km 11, Comunidad San José Km 6, Km 4 de la vía Leticia-Tarapacá, Barrio San Miguel y el Instituto de investigaciones SINCHI) (Figura 1).

\section{MATERIALES Y MÉTODOS}

Una de las principales actividades que marcan esta experiencia es el trabajo directo con el conocimiento ancestral y local. Por lo cual, se realizaron visitas a fincas y comunidades indígenas con elfin de determinar las especies más utilizadas, específicamente en la alimentación y labores de construcción de viviendas para tener en cuenta en el proyecto de banco de germoplasma. Igualmente se consultaron los lugares donde se encuentran los árboles semilleros y técnicas artesanales para el manejo de las semillas incluyendo la recolección de los frutos y temporadas de cosecha que difieren entre especies.

Finalmente, se recopiló información técnica y científica de las especies escogidas, específicamente información general de la especie, fenología, usos y experiencias de propagación de la especie.

\section{RECOLECCIÓN DE FRUTOS Y EXTRACCIÓN DE SEMILLAS}

Durante los meses de junio a octubre de 2017, se realizaron salidas de campo en búsqueda de árboles y palmas semilleras, con el fin de verificar taxonómicamente la especie, la fenología en la que se encontraban y la facilidad de recolección de los frutos. Igualmente se realizó la descripción de las semillas recolectadas y actividades de limpieza, determinación del contenido de humedad, secado, almacenamiento y pruebas de viabilidad.

\section{EXTRACCIÓN DE SEMILLA}

Los frutos recolectados fueron clasificados preliminarmente, descartando los que se encontraron con perforaciones de insectos, presencia de hongos o inmaduros. Posteriormente se dispuso a realizar la extracción de la semilla.

\section{Bactris gasipaes}

Siguiendo la metodología de extracción establecida por Peña et al. (2014) para el chontaduro, los frutos se abrieron para extraer la semilla y el fruto se utilizó con fines alimenticios. La semilla fue sumergida en agua por un día, y posteriormente se realizo el retiro total de la pulpa adherida.

\section{Socratea exhorriza}

Los frutos se obtuvieron de dos racimos diferentes de la misma palma, uno se encontraba caído sobre el suelo y el otro todavía en la palma. Los frutos que se encontraban en el suelo, en su mayoría estaban desprovistos de pulpa, mientras que los que estaban en la palma, se encontraron completos. El retiro de la pulpa se realizo con una navaja y posteriormente se realizo un lavado de las semillas. 


\section{Oenocarpus bataua}

Los frutos de milpesos fueron colectados de un racimo maduro que se encontraba en el suelo. Posteriormente se sumergieron en agua tibia y se dejaron por dos días, tiempo al cual se realizó el retiro de la pulpa, raspando suavemente el fruto hasta obtener la semilla.

\section{Carapa guianensis}

Los frutos de la andiroba fueron recolectados del suelo alrededor del árbol. Con un cuchillo se realizaron cortes al fruto para abrir la cápsula semileñosa y obtener las semillas. Se encontraron frutos abiertos con la semilla expuesta, por lo que la extracción se realizó de forma manual.

\section{Scleronema praecox}

Los frutos del castaño fueron recolectados directamente del suelo. Durante la recolección se encontraron cápsulas abiertas, lo que facilito la extracción de la semilla.

\section{LIMPIEZA Y PUREZA}

Cada muestra de semillas por especie fue marcada para el registro y pesada (semilla pura), posteriormente se separó la semilla demasiado pequeña (respecto al promedio observado), la afectada por insectos o con hongos (semilla impura). Para la determinación del porcentaje de pureza se empleo la fórmula de la guía para la manipulación de semillas forestales (Willan, 1991):

$$
\% \text { Pureza }=\frac{\text { Peso de la semilla pura }}{\text { Peso total de la muestra }} \times 100
$$

\section{CONTENIDO DE HUMEDAD (CH)}

Para conocer el porcentaje de humedad en las semillas recolectadas, se tuvo en cuenta la metodología expuesta por Willan (1991) y Rao et al. (2007) para las semillas del trópico: a. Limpieza y selección de las semillas de calidad.

b. Selección aleatoria de muestras de semillas ( 2 repeticiones de 5 semillas cada una) para determinar el contenido de humedad- $\mathrm{CH}$.

c. Pesaje de muestras de semillas (peso inicial).

d. Colocar las semillas en el horno deshidratador a $103^{\circ} \mathrm{C}$ a temperatura constante durante 17 horas

e. Pesaje de muestras de semillas (peso final).

f. Determinación del CH mediante la fórmula:

$\begin{gathered}\text { Contenido } \\ d e \\ \text { humedad }\end{gathered}=\frac{\begin{array}{c}\text { Peso } \\ \text { original }\end{array} \quad \begin{array}{c}\text { Peso tras secado } \\ \text { en estufa }\end{array}}{\text { Peso original }} \times 100$

g. Una vez determinado el $\mathrm{CH}$, se calculó el peso final de las semillas para que alcancen el $\mathrm{CH}$ deseado, que fue establecido en un rango de 9-8\%:

$\begin{gathered}\begin{array}{c}\text { Peso final } \\ \text { de las } \\ \text { semillas }\end{array} \\ \text { semo inicial } \\ \text { de las } \\ \text { semillas }\end{gathered} \times \begin{gathered}\left(\begin{array}{c}100 \text { - contenido } \\ \text { de humedad } \\ \text { inicial })\end{array}\right. \\ (100 \text { - contenido de humedad objetivo })\end{gathered}$

Para el secado las semillas, se empleó el método de secado pasivo utilizando sílica gel con indicador, en el cual se introdujeron las semillas en bolsas con cierre hermético en buen estado para controlar la humedad junto con sílica gel en proporción 3:1. Las semillas se pesaron dos veces por semana para determinar el contenido de humedad, y se regeneró la sílica a través del calor, mediante el uso de un horno desecador a $90{ }^{\circ} \mathrm{C}$ por dos horas. Después de regenerada la sílica se guardó nuevamente en las bolsas herméticas junto a las semillas.

\section{VIABILIDAD DE LAS SEMILLAS}

Para determinar la viabilidad de las semillas, se realizaron dos ensayos; el primero (Lote 1) consistió en tomar de 20 a 60 semillas por especie 
de las cinco especies seleccionadas sin secado previo, las cuales fueron sembradas en diferentes sustratos de origen orgánico dispuesto en bolsas de polietileno negro, bajo cubierta de polisombra de $50 \%$ de entrada de luz. El segundo ensayo Lote 2 , se realizó con semillas que pasaron por el proceso de secado llegando al porcentaje óptimo de contenido de humedad $( \pm 8 \%)$ para su almacenamiento. Se tomaron aleatoriamente de 50 a 80 semillas por especie y se realizó la siembra, bajo condiciones similares a las semillas del Lote 1.

Se realizaron conteos se semillas germinadas cada dos días a partir de una semana después de la siembra desde el mes de septiembre 2017 hasta diciembre 2017 para el Lote 1 y de noviembre 2017 a marzo de 2018 para el Lote 2.

\section{DISEÑO EXPERIMENTAL}

Cada muestra por especie tuvo dos réplicas, cada una con igual cantidad de semillas en dos lotes establecidos, Lote 1 corresponde a las semillas sembradas poscosecha (testigo) y Lote 2 semillas con tratamiento de secado-control $\mathrm{CH}$. Los datos registrados fueron días de germinación, total semillas germinadas (considerando como germinada al observarse el tallo con brotes foliares, total plántulas normales, plántulas anormales (crecimiento anormal de tallo, hojas), duras/dormantes (semillas en buen estado pero que al finalizar el muestreo, no germinaron), muertas (semillas con daños producidos por insectos, hongos, en estado de degradación).

\section{ANÁLISIS ESTADÍSTICO}

Se realizó utilizando el paquete Excel y el software InfoStat (versión libre) para la elaboración de bases de datos, gráficas de contenido de humedad y curvas de germinación.

\section{RESULTADOS Y DISCUSIÓN}

\section{Selección de especies y usos}

Para seleccionar las especies a estudiar en el banco de germoplasma, se tuvo en cuenta las especies

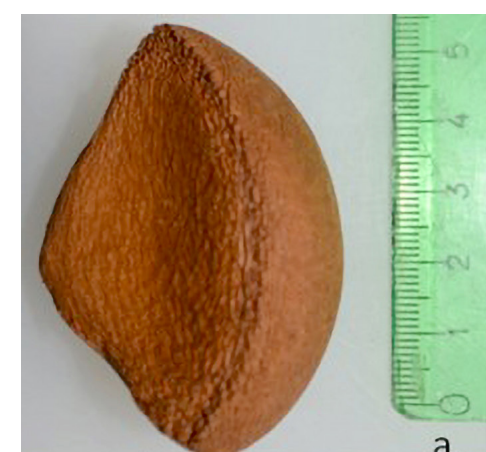

a.
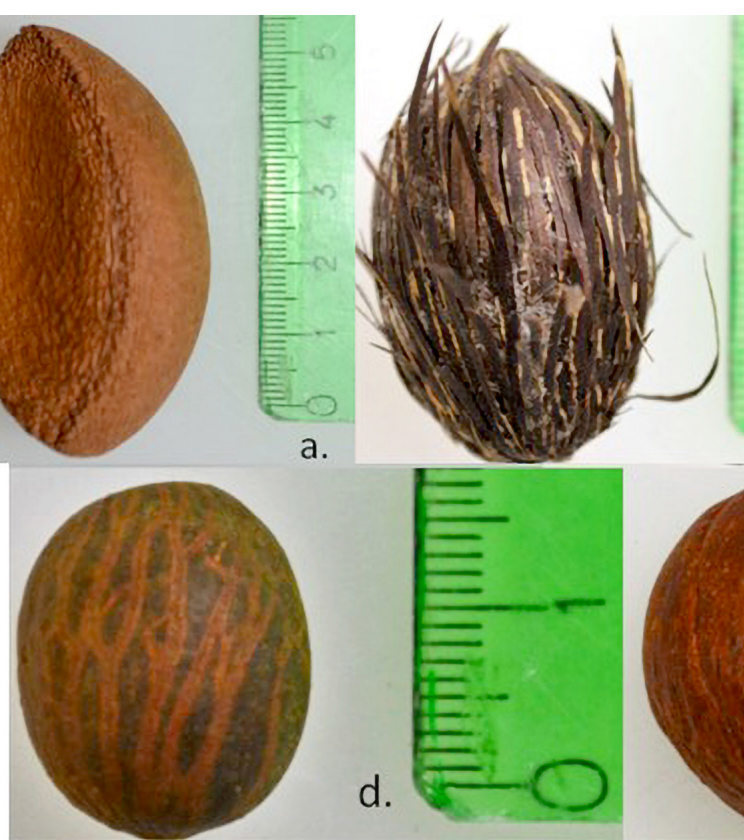

d.

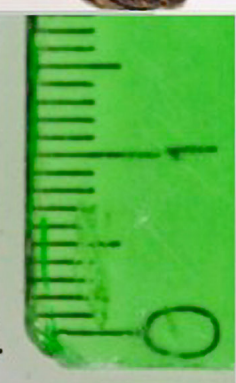

b.

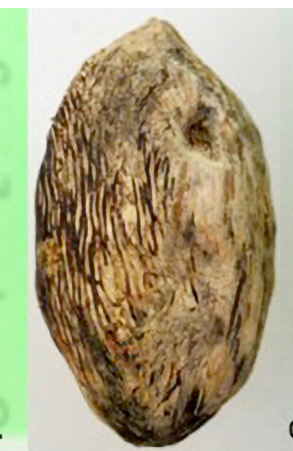

c.

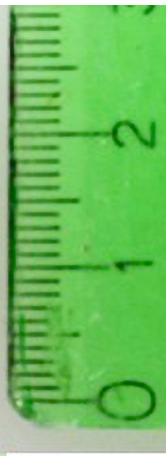

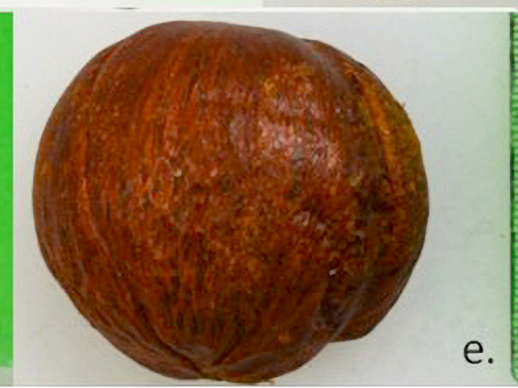

e.

Figura 2. Semillas colectadas. a. Carapa guianensis, b. Oenocarpus bataua, c. Bactris gasipaes, d. Socratea exhorriza, e. Scleronema praecox 
maderables con mayor demanda en la ciudad de Leticia y aquellas más usadas en la alimentación y construcción. Finalmente se realizó la selección de once (11) especies entre árboles y palmas de interés para el Banco de Germoplasma. Teniendo en cuenta la temporada de fructificación entre los meses de septiembre a octubre, se recolectaron frutos de cinco especies forestales nativas, Carapa guianensis, Oenocarpus bataua, Bactris gasipaes, Scleronema praecox y Socratea exorrhiza (Figura 2).

\section{CONOCIMIENTO DE ESPECIES}

El conocimiento de las especies seleccionadas fue adquirido a través de entrevistas libres y la búsqueda de información bibliográfica. Dada la cosmovisión que tienen las comunidades indígenas para el manejo Tabla 1. Calendario ecológico de cinco (5) especies florísticas

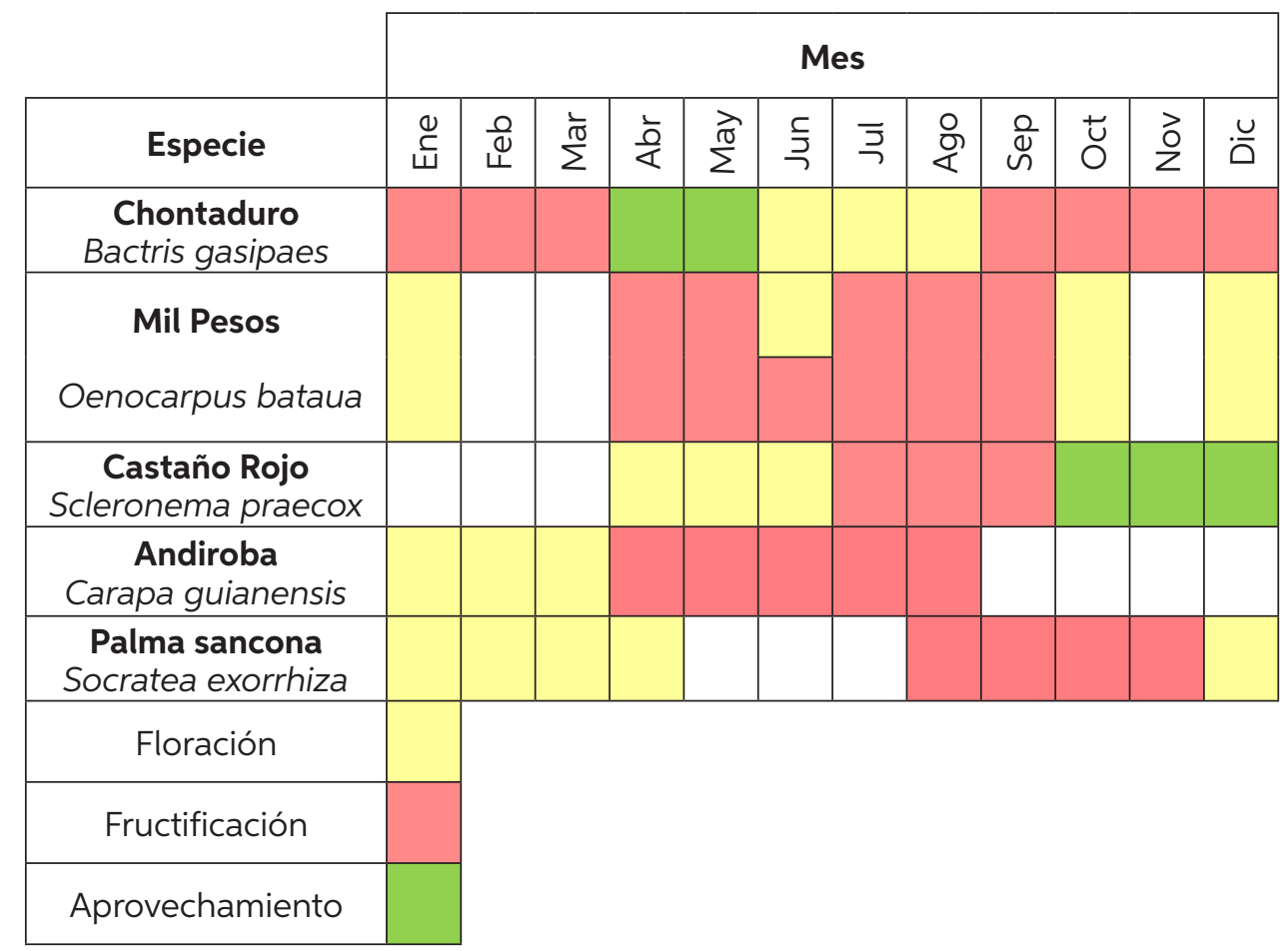

del bosque, es importante tener en cuenta ese conocimiento ancestral para el manejo de plántulas y semillas de las especies seleccionadas y poder aplicar las técnicas correspondientes con el fin de conservar el germoplasma.

El primer dato obtenido corresponde a las diferentes temporadas de cosecha que tienen estas especies, cómo se propagan naturalmente y cómo poder conservar el germoplasma. Finalmente se recopiló la información en un calendario ecológico (Tabla 1) que demarca la fenología de floración y fructificación de las especies seleccionadas, además de identificar la época en la que se realiza el aprovechamiento en Leticia.
Cabe resaltar en esta experiencia, el aprovechamiento que se da a la palma del chontaduro, en la cual, la segunda cosecha del año es la principal (enero a marzo) y la que más se aprovecha para consumo o para la venta. La primera cosecha es conocida como cosecha

de animales (septiembre a diciembre) y su aprovechamiento es bajo.

\section{EXPERIENCIAS DE PROPAGACIÓN}

El manejo de las especies forestales por parte de las comunidades indígenas se ha realizado con base a la observación y enseñanzas que pasan de generación en generación según lo indicado por los entrevistados.

La información recogida indica las temporadas de aprovechamiento, usos, prácticas de cultivo. Sin embargo, cabe destacar que la información de propagación por parte de las comunidades es poca, debido a que la regeneración y propagación es natural. A continuación se recopila la siguiente 
Tabla 2. Conocimiento local de experiencias de propagación de especies amazónicas en Leticia

\begin{tabular}{|l|l|}
\hline Especie & Experiencia \\
\hline Bactris gasipaes & $\begin{array}{l}\text { No se obtuvieron experiencias de propagación de chontaduro por parte de las } \\
\text { personas encuestadas. Es un fruto muy comercializado para el consumo. }\end{array}$ \\
\hline Oenocarpus bataua & $\begin{array}{l}\text { Los frutos de esta palma son recolectados para extraer aceite o realizar bebidas. } \\
\text { Primero maduran el fruto en agua tibia y luego manipulan la semilla dependiendo } \\
\text { del uso deseado. Las semillas son escarificadas como resultado de la manipulación y } \\
\text { dispersadas en alrededores de la vivienda o área de cultivo tradicional. }\end{array}$ \\
\hline Scleronema praecox & Trasplantan las plántulas que crecen alrededor del árbol semillero \\
\hline Socratea exorrhiza & $\begin{array}{l}\text { En Leticia no se reportan experiencias de propagación de esta especie. Utilizada para } \\
\text { construcciones de viviendas. }\end{array}$ \\
\hline Carapa guianensis & En Leticia no se reportan experiencias de propagación de esta especie \\
\hline
\end{tabular}

Tabla 3. Valores registrados en las actividades del Banco de germoplasma de cinco especies amazónicas

\begin{tabular}{|c|c|c|c|c|c|}
\hline Especie & Procedencia & $\% \mathrm{CH}$ & $\%$ Pureza & $\begin{array}{c}\text { Peso } \\
\text { inicial }\end{array}$ & CH Mínimo alcanzado \\
\hline Bactris gasipaes & Comunidad Nazareth & 51.71 & 98.1 & 219.3 & \multirow{3}{*}{7.92} \\
\hline Bactris gasipaes & Comunidad San José & 51.71 & 90.0 & 45.0 & \\
\hline Bactris gasipaes & Comunidad Casillanaira & 51.71 & 88.9 & 40.6 & \\
\hline Carapa guianensis & Instituto Sinchi & 43.07 & 94.4 & 2210 & 9.58 \\
\hline Socratea exorrhiza & Comunidad Nazareth & 35.96 & 94.3 & 295.2 & 7.78 \\
\hline Oenocarpus bataua & Comunidad Nazareth & 29.54 & 68.0 & 797.2 & 9.41 \\
\hline Scleronema praecox & Comunidad Bora Km 17 & 59.55 & 89.94 & 5375 & 52.20 \\
\hline
\end{tabular}

información de experiencias de propagación (Tabla 2).

\section{RECOLECCIÓN DE FRUTOS Y MANEJO DE SEMILLAS}

Una vez determinadas las especies a colectar, se realizaron salidas de campo para la búsqueda y localización de árboles y palmas semilleras. Lo primero que se realizó, fue la determinación de la especie y posteriormente el estado fitosanitario del individuo. Se diligenciaron fichas de recolección de semillas con información taxonómica, de hábitat, localización geográfica, datos del recolector y topografía.

El material colectado fue limpiado cuidadosamente, y se determinó el porcentaje de pureza de cada muestra. Posteriormente se procedió a un secado previo a temperatura ambiente, lejos del sol directo y en lugares aireados. Una vez registradas las semillas colectadas, se tomaron 2 muestras por accesión para la determinación del contenido de humedad (Tabla 3). 

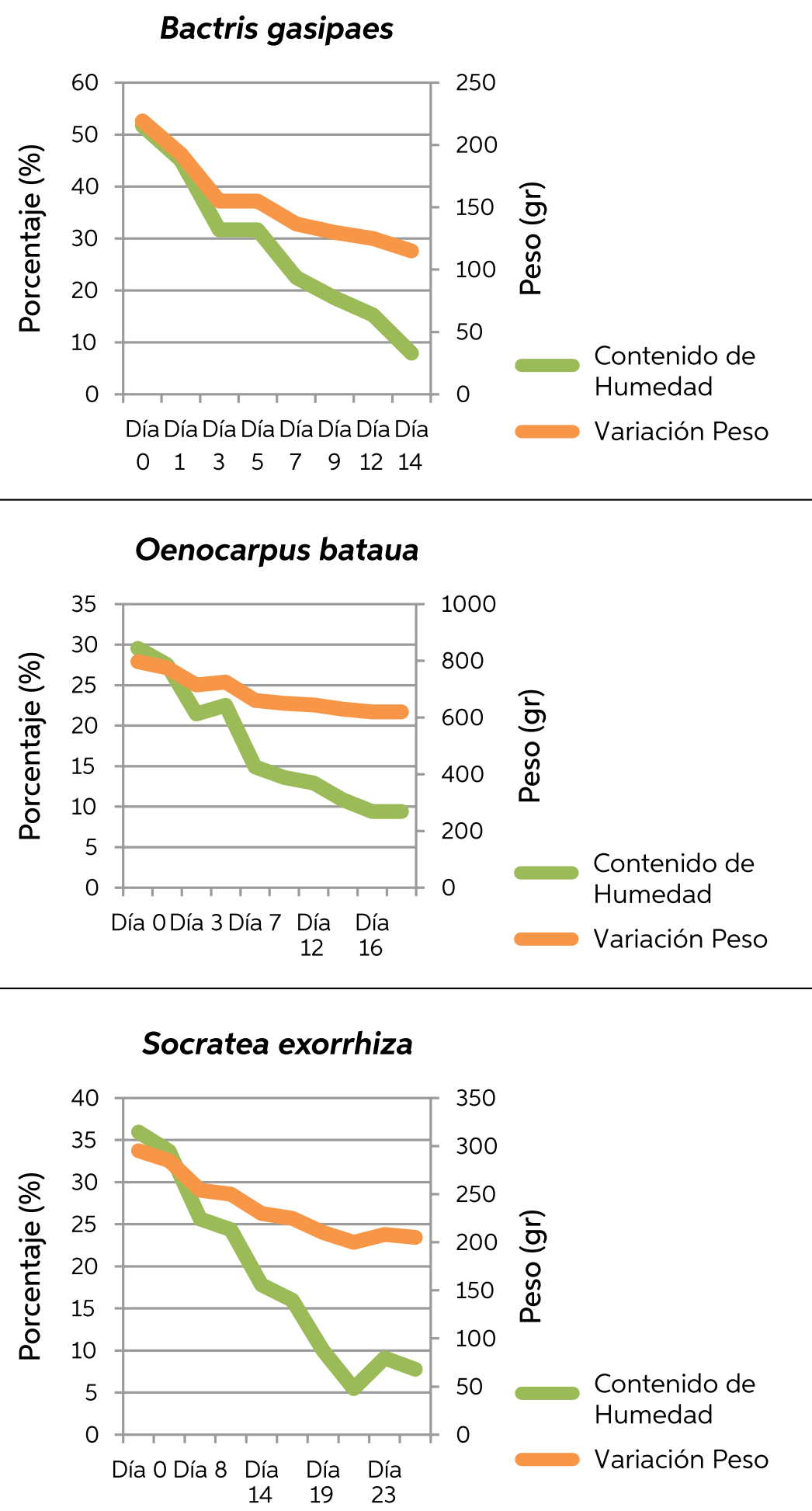

Figura 3. Variación del contenido de humedad de semillas de palma: Bactris gasipaes, Oenocarpus bataua y Socratea exhorriza durante el secado.

\section{VARIACIÓN DEL CONTENIDO DE HUMEDAD}

\section{Chontaduro (Bactris gasipaes)}

Las semillas almacenadas de chontaduro, presentaron variaciones de contenido de humedad y peso desde el inicio del secado hasta su finalización y almacenamiento.

Las semillas fueron almacenadas cuando alcanzaron el $8 \%$ de contenido de humedad a los 20 días de secado, posteriormente, el peso de la muestra se mantuvo constante (Figura 3).

De acuerdo a Peña et al., (2014), las semillas de chontaduro almacenadas por períodos de tiempo superior a cuatro meses, pueden disminuir el porcentaje germinativo, por esta razón, todas las semillas almacenadas iniciaron las pruebas de germinación en vivero.

\section{Palma mil pesos (Oenocarpus bataua)}

La variación del peso y contenido de humedad de la palma milpesos, fue constante desde el inicio del secado hasta su almacenamiento, donde alcanzó un $9.4 \%$ de contenido de humedad (Figura 3). No se presentó contaminación por hongos, ni ataque de insectos.

En cuanto a las palmas, el chontaduro (Bactris gasipaes) tuvo un contenido de humedad alto en comparación con las otras especies de palma, sin embargo, reaccionó positivamente al secado y almacenamiento. La palma de milpesos presentó el menor 
porcentaje de $\mathrm{CH}$ con el $29,54 \%$ con resultados positivos en el secado y almacenamiento. La palma zancona, obtuvo un CH $36 \%$ y fue secada y almacenada sin complicaciones.

\section{Andiroba (Carapa guianensis)}

La variación del contenido de humedad y peso en la muestra de semillas de la andiroba fue acelerada durante las primeras semanas y posteriormente fue paulatinamente disminuyendo hasta que se alcanzó un peso estable (Figura 4).

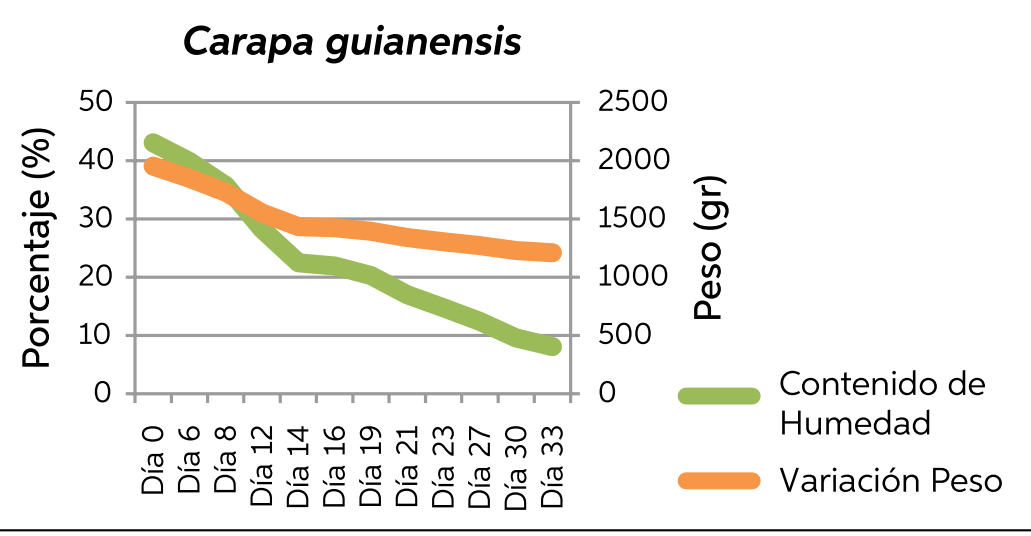

Figura 4. Variación del contenido de humedad de semillas de Carapa guianensis durante el secado.

Las semillas de andiroba recién recolectadas presentaron un contenido de humedad de $43 \%$, y durante el mantenimiento de las semillas en bolsas plásticas con sílica gel alcanzaron un $\mathrm{CH}$ estimado de 8\%. De acuerdo a Ferraz \& Sampaio (1996) en pruebas de almacenamiento con semillas de andiroba en Manaos, las semillas deben estar en contenidos de humedad de 90 a 87.5, debido a que si superan la deshidratación hasta el $50.4 \%$, se da pérdida de viabilidad, sin embargo, en este estudio, las semillas colectadas presentaron un bajo contenido de humedad (43\%) respecto al estudio citado y presentaron una germinación de 79.2\%. Se observó que al disminuir este valor de $\mathrm{CH}$, se presenta una total pérdida de viabilidad (ver resultados viabilidad semillas). Estas variaciones en el contenido de humedad de las semillas de andiroba, pueden obedecer a cambios físicos dados por la región y el tipo de ecosistema en el que se encontraban los árboles.

Durante el secado y almacenamiento, la muestra fue afectada por larvas del insecto de las meliáceas (Hypsipyla sp.) y contaminada por hongos a causa de daños en la bolsa hermética, situación que también se ha presentado en estudios anteriores (Ferraz \& Sampaio, 1996). Se realizó depuración de la muestra, mejoramiento en el almacenamiento, logrando un mantenimiento de la calidad de las semillas en buen estado.

Frente al ataque de la larva de insecto a los frutos de andiroba y teniendo en cuenta las condiciones de almacenamiento, es posible que éstos fueran infestados antes de la recolección del fruto, por lo que sería indispensable, realizar una revisión exhaustiva de presencia de microorificios en la semilla durante el secado y antes de realizar el almacenamiento o utilizar el método deinmersión señalado por Ferraz \& Sampaio (1996).

\section{Castaño rojo (Scleronema praecox)}

La variación del contenido de humedad del castaño rojo solamente se pudo monitorear durante una semana, debido a que se presentaron problemas con la integridad de la semilla por desecación y hongos.

El porcentaje de $\mathrm{CH}$ permite realizar un adecuado secado de las semillas que permitirá su almacenamiento conservando la viabilidad de la semilla. Altos porcentajes de humedad, presentan inconvenientes en el secado o almacenamiento, como ocurrió con las semillas de castaño rojo (Scleronema praecox) que presentaron 
el porcentaje de CH más alto (59,54\%) y que durante el secado presentó contaminación por hongos y pérdida de integridad de la semilla (desecación rápida).

En general, se recomienda manejar la semilla de Scleronema praecox como recalcitrante, debido a que exhibe sensibilidad a la desecación, se dispersa junto con los tejidos del fruto que es carnoso (observación en campo) y presentan altos contenidos de humedad (Doria, 2010), por lo cual las actividades de conservación se limitan al establecimiento de plántulas en vivero.

\section{VIABILIDAD DE LAS SEMILLAS}

En total se evaluaron dos lotes experimentales para determinar la viabilidad de las semillas recolectadas. En la tabla 4 se detalla información respecto a la siembra de las semillas. El primer lote (Lote 1) corresponde a las semillas que se sembraron después de recolectadas, el segundo lote (Lote 2) con las semillas que se sometieron a secado hasta alcanzar un $\mathrm{CH}$ estable. Para determinar la viabilidad de las semillas, se realizaron los cálculos expuestos en Willan (1991).

El porcentaje de germinación se obtuvo del total de semillas germinadas frente al total de semillas sembradas, el período de energía como la cantidad de días requeridos para alcanzar el valor máximo de germinación. La energía de germinación se calculo teniendo en cuenta el período de energía hasta que la germinación

Tabla 4. Tratamientos pregerminativos y sustratos utilizados para siembra de semillas de cinco especies amazónicas.

\begin{tabular}{|c|c|c|c|}
\hline Lote experimental 1 & No. Sembradas & $\begin{array}{l}\text { Tratamiento } \\
\text { pregerminativo }\end{array}$ & Sustrato \\
\hline Socratea exhorriza & 20 & No & $\begin{array}{l}\text { Arena, gallinaza, lombricompuesto } \\
\qquad 1: 1: 1\end{array}$ \\
\hline Oenocarpus bataua & 20 & $\begin{array}{c}\text { Imbibición en agua tibia } \\
\text { y escarificación }\end{array}$ & $\begin{array}{c}\text { Compost-tierra negra } \\
1: 1\end{array}$ \\
\hline Bactris gasipaes & 20 & No & $\begin{array}{c}\text { Compost-tierra negra } \\
1: 1\end{array}$ \\
\hline Scleronema praecox & 60 & No & $\begin{array}{l}\text { Arena, gallinaza, tierra negra } \\
\qquad 1: 1: 1\end{array}$ \\
\hline Carapa guianensis & 48 & No & $\begin{array}{l}\text { Arena, gallinaza, tierra negra } \\
\qquad 1: 1: 1\end{array}$ \\
\hline Lote experimental 2 & No. Sembradas & $\begin{array}{l}\text { Tratamiento } \\
\text { pregerminativo }\end{array}$ & Sustrato \\
\hline Socratea exhorriza & 50 & $\begin{array}{c}\text { Imbibición por } 15 \\
\text { minutos y escarificación }\end{array}$ & Aserrín maduro \\
\hline Oenocarpus bataua & 50 & $\begin{array}{l}\text { Imbibición por } 15 \\
\text { minutos }\end{array}$ & $\begin{array}{l}\text { Lombricompost, tierra negra } \\
\qquad 1: 1\end{array}$ \\
\hline Bactris gasipaes & 80 & $\begin{array}{l}\text { Estimulación en cloro al } \\
6 \% \text { por } 3 \text { min, enjuague } \\
\text { y secado previo }\end{array}$ & $\begin{array}{c}\text { Tierra negra, arena } \\
1: 1\end{array}$ \\
\hline Carapa guianensis & 50 & $\begin{array}{l}\text { Imbibición por } 15 \\
\text { minutos }\end{array}$ & $\begin{array}{c}\text { Tierra negra, arena } \\
1: 1\end{array}$ \\
\hline
\end{tabular}


Tabla 5. Resultados de las pruebas de viabilidad a las semillas recolectadas

\begin{tabular}{|c|c|c|c|c|c|c|}
\hline \multirow[b]{2}{*}{ ESPECIE } & \multicolumn{3}{|c|}{ Lote 1} & \multicolumn{3}{|c|}{ Lote 2} \\
\hline & ○。 & 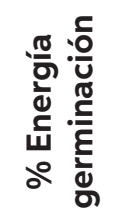 & 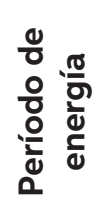 & ○。 & 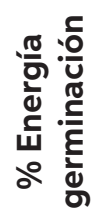 & 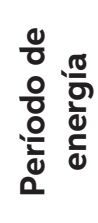 \\
\hline Socratea exorrhiza & 25,00 & 15,00 & 92 & - & - & - \\
\hline Carapa guianensis & 79,17 & 41,67 & 25 & - & - & - \\
\hline Scleronema praecox & 89,55 & 40,30 & 14 & - & - & - \\
\hline Bactris gasipaes & 20,00 & 5,00 & 89 & - & - & - \\
\hline Oenocarpus bataua & 80,00 & 80,00 & 43 & - & - & - \\
\hline
\end{tabular}

\section{Curvas de germinación}

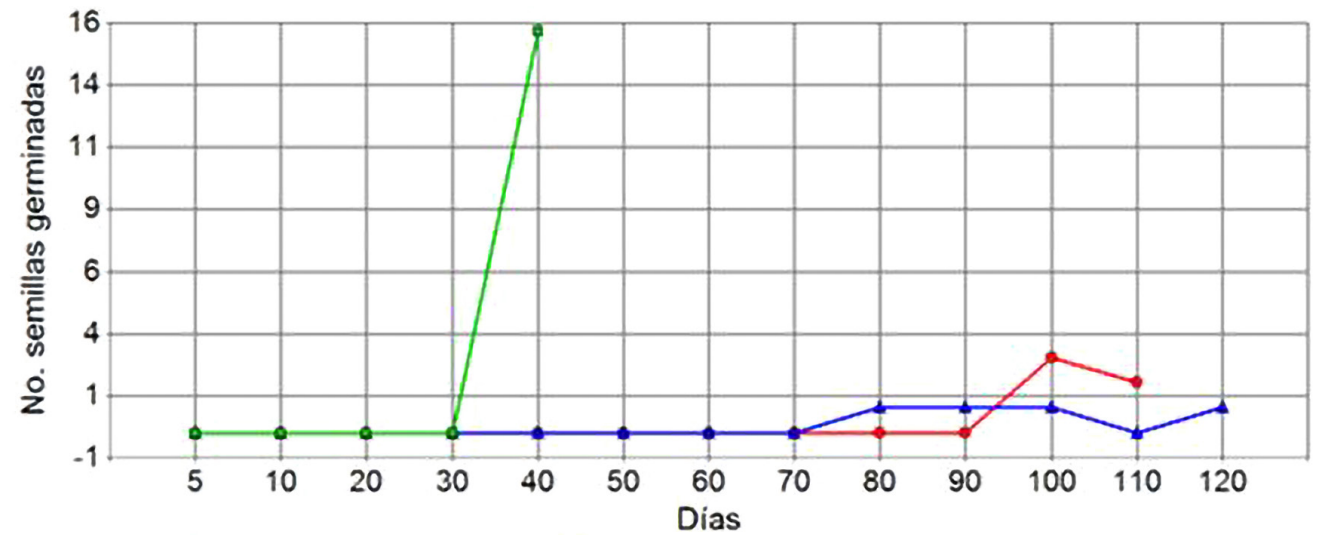

$\rightarrow$ Socratea exorrhiza $\rightarrow$ Bactris gasipaes $\rightarrow$ - Oenocarpus bataua

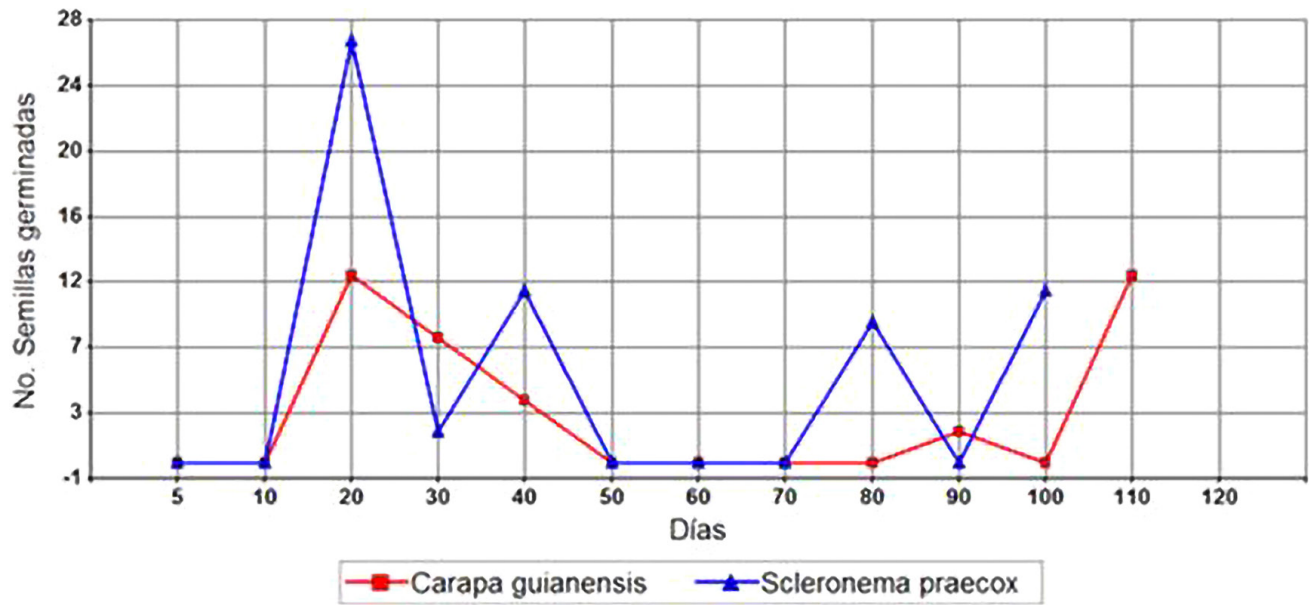

Figura 5. Curvas de germinación del Lote 1. El primer gráfico corresponde a especies maderables y el segundo gráfico a especies de palmas. 
descendió del valor máximo de germinación, razón por la cual, el ensayo duró mas de tres meses.

El primer lote experimental presentó semillas viables, mientras que el lote dos, no presentó germinación (Tabla 5). Las especies maderables presentaron menor período de latencia y mayor porcentaje de germinación frente a las especies de palmas, que tuvieron períodos muy largos de latencia y baja germinación (Figura 5).

Las especies Scleronema praecox, Oenocarpus bataua y Carapa guianensis presentaron los mayores porcentajes de germinación (89.55\%, $80 \%$ y $79,2 \%$ respectivamente), en comparación con las palmas Socratea exorrhiza y Bactris gasipaes (25\% y $20 \%)$. En cuanto a la energía germinativa, la especie que presento mejor desempeño germinativo fue la palma Oenocarpus bataua, debido a que la mayoría de las semillas germinaron en un solo período de energía. Para obtener las semillas de mil pesos se realizo el proceso tradicional que consiste en sumergir los frutos con agua caliente por un tiempo entre 10 y 15 minutos y luego realizar la escarificación manual.

Las especies de chontaduro y palma zancona presentaron bajos porcentajes de energía de germinación, que puede obedecer a la poca selectividad de los frutos obtenidos. En el caso de la zancona, según los resultados obtenidos por Potvin et al. (2003), las semillas de Socratea exorrhiza recogidas del suelo se encuentran maduras y aptas para la germinación, mientras que las semillas obtenidas para el estudio se recolectaron directamente del racimo en la palma.

En el caso del chontaduro, el período de germinación estuvo entre 89-111 días, resultados similares a los reportados por Ferreira \& Santos (1992). Teniendo en cuenta el conocimiento tradicional, el bajo porcentaje de germinación de las semillas de chontaduro puede obedecer a la temporada de colecta, debido a que la mejor cosecha de frutos se realiza en el primer período del año y la recolección se realizó en el segundo semestre del año, donde se produce la denominada cosecha de animales.

El castaño rojo presento un porcentaje de energía de germinación del $40.30 \%$ en un período de energía de 14 días, el período más corto en presentarse germinación. Esta especie se estableció fácilmente en el vivero y no requirió tratamiento pregerminativo.

La andiroba presentó un porcentaje de energía germinativa de $41.67 \%$, las semillas germinaron de forma gradual, registrandosé plantulas con crecimiento rápido y otras, con un crecimiento más lento. No se realizo tratamiento pregerminativo a las semillas.

Respecto al segundo lote experimental, no hubo resultado positivo para ninguna de las muestras.

En el caso de la andiroba (Carapa guianensis), la deshidratación de las semillas por debajo del 43\% causó una total pérdida de viabilidad, tal como lo registrado por Ferraz \& Sampaio (1996), quienes afirman que al aumentar la deshidratación, se reduce la viabilidad de las semillas de andiroba, por lo que se pueden considerar como recalcitrantes.

La palma zancona (Socratea exorrhiza) no presentó ninguna germinación, a pesar que se siguió la metodología desarrollada por Potvin et al. (2003) y Bocanegra (2010). Sin embargo existen dos variables que pudieron afectar la germinación de las semillas, primero, los frutos se colectaron directamente del racimo en la palma y segundo, el secado de las semillas pudo haber afectado la viabilidad de las semillas.

De las semillas de chontaduro (Bactris gasipaes) que se sometieron a disminución del contenido de humedad, ninguna presento resultados positivos, reafirmando los resultados de Ferreira \& Santos (1992), Ferreira \& Santos 
(1993), Carvalho \& Muller (1998) quienes demostraron que al disminuir los porcentajes de contenido de humedad, disminuye el porcentaje de germinación de las semillas, calificando las semillas de chontaduro como recalcitrantes.

Las semillas de Oenocarpus bataua con contenido de humedad de $8 \%$ y sembradas en el vivero, no presentaron germinación. De acuerdo a Nazário \& Ferreira (2012), las semillas de milpesos son susceptibles a la deshidratación, razón por la cual, son semillas recalcitrantes, y a mayor cantidad de humedad, mejor resultados presentan en la germinación.

En general, en esta investigación se confirman los resultados generados por la pérdida de humedad de las semillas en el comportamiento germinativo de las mismas, siendo vulnerables a la desecación. Por esta razón, se recomienda manejar las semillas con altos niveles de humedad en el banco de germoplasma, realizar almacenamiento de corto plazo y continuar con la propagación en vivero de las especies amazónicas, debido a que presentaron resultados positivos en los experimentos de germinación.

\section{CONCLUSIONES}

El presente estudio incluyó la colecta, limpieza, pureza, contenido de humedad y germinación de semillas de cinco especies vegetales amazónicas, de mucho interés para la comunidad por sus variados usos como el consumo humano o construcción, generando una fuerte presión en las poblaciones de estas especies en el municipio de Leticia y de las cuales no se tienen mayores referencias de propagación en la región.

Teniendo en cuenta los resultados obtenidos, el banco de germoplasma en el municipio de Leticia, debe ser sin almacenamiento de semillas, es decir, un banco germoplasma de campo, realizando la propagación y mantenimiento las especies vivas tipo vivero, debido a que muchas de las semillas de especies vegetales tropicales son consideradas como semillas recalcitrantes.

\section{AGRADECIMENTOS}

Este trabajo se realiza gracias al apoyo financiero de Sennova en el marco del proyecto “Conservación, preservación y protección de las Semillas del Amazonas, mediante la creación de un Banco de germoplasma, para garantizar de manera sostenible la biodiversidad y la seguridad alimentaria en la región" ejecutado en el SENACentro para la Biodiversidad y el Turismo del Amazonas.

Agradecemos a las comunidades indígenas de Nazareth, San José (km 6), Casillanaira (km 11), comunidad Bora ( $\mathrm{km} \mathrm{17)}$ ) por permitir la entrada a sus territorios para la recolección de información y semillas. Igualmente al personal que participó en la recolección de información de especies, semillas y manejo del vivero Nelson Cayetano, Víctor Perdomo, Juana Parente, Numa Castro, Evis Braga.

\section{BIBLIOGRAFIA CITADA}

Alcaldía de Leticia. 2002. Plan básico de ordenamiento territorial. Alcaldía de Leticia, Colombia. 85 pp.

Alcaldía de Leticia. 2018. Nuestro municipio (http://www.leticia-amazonas.gov.co/ municipio/nuestro-municipio). Acceso: 20/04/2018

Bocanegra, Lourdes. 2010. Ensayo de germinación de Socratea exorrhiza (Martius) Wendland, con cuatro sustratos y dos tipo de tinglado. Tesis de pregrado, Universidad Nacional de la Amazonía Peruana, Facultad de ciencias forestales, Iquitos, Perú. 83 pp. 
Cárdenas, D.; Castaño, N.; Sua, S.; Quintero, L. 2015. Planes de manejo para la Conservación de Abarco, Caoba, Cedro, Palorosa y Canelo de los Andaquíes. (https://www.sinchi.org.co/ planes-de-manejo-para-la-conservacion-deabarco-caoba-cedro-palorosa-y-canelo-delos-andaquies). Acceso: 01/03/2017

Carvalho, J.E.; Muller, C.H. 1998. Níveis de tolerância e letal de umidade em sementes de pupunheira, Bactris gasipaes. Revista brasileira de fruticultura, 20(3): 283-289.

Doria, J.2010. Generalidades sobre las semillas: su producción, conservación y almacenamiento. Cultivos tropicales, 31(1): 74-85.

Ferraz, I. D.; Sampaio, P. 1996. Métodos simples de armazenamento das sementes de andiroba (Carapa guianensis Aubl. e Carapa procera D.C.-Meliaceae). Acta amazónica, 26(3): 137144.

Ferreira, S.A.; Santos, L.A. 1993. Efeito da velocidade de secagem sobre emergência e vigor de sementes de pupunha (Bactris gasipaes Kunth). Acta amazonica, 23(1): 3-8.

Ferreira, S.A.; Santos, L.A. 1992. Viabilidade de sementes de pupunha (Bactris gasipaes) Kunth. Acta amazonica, 22(3): 303-3

Nazário, P.; Ferreira, S.A. (2012). Emergência de plântulas de patauá (Oenocarpus bataua
Mart.) em função do dessecamento das sementes. Informativo Abrates, 22(1), 22-24.

Peña, E.; Tolosa, W.; Reyes, H.M.; Quintero, É.M.; Jaramillo, A.; Carabalí, A. 2014. Manejo técnico para el establecimiento de vivero de chontaduro (Bactris gasipaes Kunth): boletín técnico. (https://www.javerianacali.edu.co/ sites/ujc/files/node/field_documents/field_ doc). Acceso: 29/09/2017

Potvin, C.; Cansari, R.; Hutton, J.; Caisamo, I.; Pacheco, B. 2003. Preparation for propagation: understanding germinatio of giwa (Astrocaryum standletanum), wagara (Sabal maurittiformis), and eba (Socratea exorrhiza) for future cultivation. Biodiversity and Conservation, 12: 2161-2171.

Rao, N.K.; Hanson, J.; Dullo, M.E.; Ghosh, K.; Novell, D.; Larinde, M. 2007. Manual para el manejo de semillas en bancos de germoplasma. Manuales para Bancos de Germoplasma No. 8. (https:// www.bioversityinternational.org/fileadmin/ user_upload/online_library/publications / pdfs/1261.pdf). Acceso: 06/09/2017

Willan, R.L. 1991. Guía para la manipulación de semillas forestales. (http://www.fao.org/ docrep/006/AD232S/AD232S00.HTM). Acceso: 29/08/2017

Recibido: 23 de abril de 2018 Aceptado para publicación: 30 de mayo de 2018 